\title{
Measuring the plasma composition in tokamaks with metallic plasma-facing components
}

\author{
M. Sertoli ${ }^{1,2} \dagger$, P. Carvalho ${ }^{3}$, C. Giroud ${ }^{2}$, S. Menmuir ${ }^{2}$ and JET \\ Contributors $\ddagger$ \\ ${ }^{1}$ Max-Planck-Institut für Plasmaphysik, Boltzmannstrasse 2, D-85748 Garching, Germany \\ ${ }^{2}$ CCFE, Culham Science Centre, Abingdon, Oxon, OX14 3DB, UK \\ ${ }^{3}$ Instituto de Plasmas e Fusao Nuclear, Instituto Superior Tecnico, Universidade de Lisboa, \\ Av. Rovisco Pais, 1049-001 Lisboa, Portugal
}

(Received xx; revised xx; accepted xx)

In present and future magnetic confined fusion devices with metallic plasma facing components (PFCs) such as JET-ILW and ITER, the calculation of the plasma composition must account for multiple impurities of a wide range of mass and charge, resolve their poloidal asymmetries and account for different central peaking for various elements. Single measurements of radiation and effective charge are not enough to characterized this complex system and a self-consistent analysis of data from multiple diagnostics is required. This contribution describes a method to calculate the plasma composition simultaneously accounting for contributions of up to two low-Z impurities, and two mid/high-Z impurities. The analysis stems from methodologies explained in Sertoli et al. (2018), expanded to include more impurities and to coherently analyse multiple diagnostics within the same framework. The example Ne-seeded JET-ILW hybrid discharge reported here shows that $\mathrm{Be}, \mathrm{Ne}, \mathrm{Ni}$ and $\mathrm{W}$ are necessary to simultaneously explain the observed soft X-ray emission, the $\mathrm{W}$ concentration measured by passive vacuumultra-violet spectroscopy, the line-of-sight integrated measurement of the effective charge, the observed poloidal asymmetry of the SXR emission, the Ne density measured by charge-exchange-recombination-spectroscopy and the line-of-sight integrals of the total radiation as measured by bolometry. This consistent picture of the elemental composition enables the calculation of the radial profiles of the effective charge, the dilution and total radiation. For the cases analysed up to now, these are often very different from the typical assumptions presently used when modeling JET-ILW discharges. This will affect, among others, the calculation of neutron rates, current density profile and heat transport. These considerations are of course valid for all present and future magnetic controlled fusion devices which exhibit multi-material plasma-facing components, including ITER.

\section{Introduction}

The knowledge of plasma composition impacts the estimation of many relevant quantities in magnetic-controlled-fusion plasmas. Minority species, both hydrogenic and impurity, can strongly affect the absorption of ion-cyclotron-resonance-frequency (ICRF)

$\ddagger$ See the author list of "Overview of the JET preparation for Deuterium-Tritium Operation" by E. Joffrin et al. to be published in Nuclear Fusion Special issue: overview and summary reports from the 27th Fusion Energy Conference (Ahmedabad, India, 22-27 October 2018)

$\dagger$ Email address for correspondence: marco.sertoli@ukaea.uk 
heating, the deposition profile of neutral-beam-injection (NBI), the shape of the current density profile as well as the fusion rate. Over the decades, various spectroscopic diagnostics and analysis methodologies have been developed to estimate the impurity densities and plasma effective charge (see e.g. McDermott et al. (2018); Sertoli et al. (2018); Odstrcil et al. (2018); Coenen et al. (2013); Pütterich et al. (2012); Reinke et al. (2012); Czarnecka et al. (2011); Rathgeber et al. (2010); Behringer et al. (1986) and reference therein) and on these results depend the conclusions drawn by complex analysis and modeling of present experiments as well as predictions for future machines such as ITER or DEMO (see e.g. Breton et al. (2018a); Loarte et al. $(2015)$; Beurskens et al. (2013); Zagórski et al. (2013); Kallenbach et al. (2013); Tardini et al. (2012); DelgadoAparicio et al. (2011); Lerche et al. (2011); Pacher et al. (2007); Kallenbach et al. (2005); Dux \& Peeters (2000)). These diagnostics and analysis methodologies each typically give information on one impurity element at a time, usually of one single ionization stage. They are often line-of-sight (LoS) integrals over one single chord and are in general analysed independently. In tokamaks such as ITER and JET with the ITER-Like Wall (ILW) with multi-elemental plasma-facing components (PFCs) including low- (Be), mid$(\mathrm{Ni}, \mathrm{Fe}, \mathrm{Cr}, \mathrm{Cu})$ and high-Z materials $(\mathrm{Mo}, \mathrm{W})$, as well seeding gases (e.g. N, Ne), the problem of providing a clear and coherent picture of the plasma composition is thus an arduous, but critical task. This can lead to a better understanding of the physics and to gain confidence or falsify available models, as well as give more precise indications on the impurities responsible for performance degradation or disruptions. In all the above cases, a correct identification of the impurity specie can provide vital information to improve its control, while inappropriately holding one element responsible may lead to erroneous and futile actions.

The method described in this contribution aims at providing a coherent, integrated picture of the plasma composition by combining as much information as possible from various diagnostics. Fo the case of JET-ILW these include (fig. 1):

- SXR radiation: SXR-diode array camera V (Sertoli et al. 2018$)$, vertical view, 35 LoS, $250 \mu \mathrm{m}$ Be-filter, integrated radiated power in photon energy range $1.5<E_{\gamma}<10(\mathrm{keV})$.

- Total radiation: foil bolometer arrays (Huber et al. 2007), cameras V and H, 14 vertical and horizontal LoS not viewing the divetor.

- W concentration: grazing-incidence VUV spectrometer viewing in a small wavelength range close to $5 \mathrm{~nm}$ along one vertical LoS (Schwob et al. 1987) providing two independent measurements localized close to $T_{e} \sim 1.5 \mathrm{keV}$ and $T_{e} \sim 3.5 \mathrm{keV}$ (Pütterich et al. 2008).

- Effective charge $Z_{\text {eff }}$ : spectroscopy of visible Bremmsstrahlung along one horizontal and one vertical LoS (Behringer et al. 1986).

- Toroidal rotation and ion temperature: charge-exchange-recombination-spectroscopy $(\mathrm{CXRS})$ measurements at $\sim 10$ radial positions close to the low-field-side (LFS) midplane (Menmuir et al. 2014).

- Electron temperature and density: Thomson scattering (HRTS) measurements at $\sim 60$ radial positions close to the LFS midplane (Pasqualotto et al. 2004 , Frassinetti et al. 2012).

Additional information can also be included for the following quantities: electron density and temperature measurements from LIDAR Thomson Scattering (Maslov et al. 2013) or electron cyclotron emission diagnostics (de la Luna et al. 2004); Zeff measurement along the vertical LoS of visible spectroscopy measurements; Ni concentration measurement from the X-ray crystal spectrometer measuring He-like Ni (Shumack et al. 2014); information on the impurity content from other UV spectrometers; toroidal rotation 


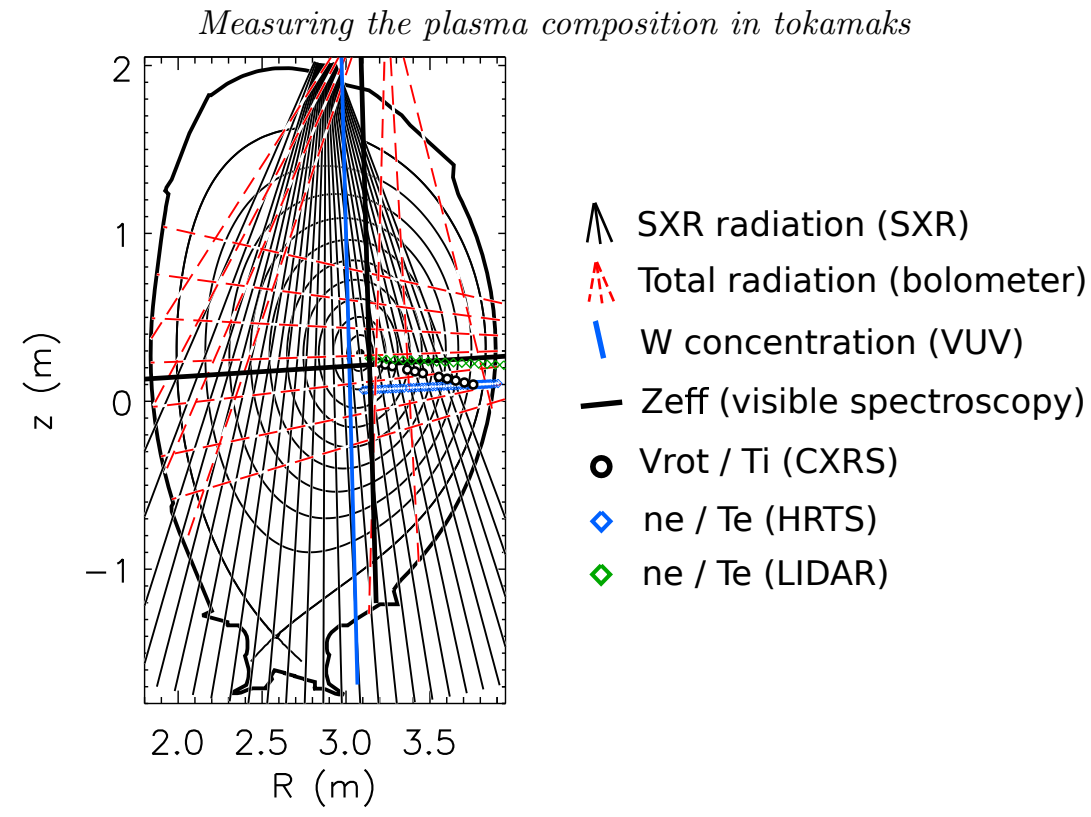

FiguRE 1. Lines of sight and measurement positions of the diagnostics included in the integrated data analysis.

estimate the X-ray crystal spectrometer or MHD markers. All of these diagnostics are analysed on the same EFIT equilibrium reconstruction (Lao et al. 1985) performed using only magnetic measurements, accounting for possible corrections identically for all measurement positions and LoS.

An overview of the whole method will be given in section 2, concentrating mainly on the most recent extensions to account for multiple low-Z and medium-/high-Z elements. In section 3 a case discharge will be analysed, describing in-depth the stepwise inclusion of multiple impurities searching for the best match to all the available diagnostic measurements. Discussion on the result, conclusions and an outlook are given in section 4

\section{Experimental method}

The extension of the method starts with a generalization of equation (4) from Sertoli et al. (2018) for any impurity specie (not just tungsten) of atomic number $Z_{0}$ dominating the SXR diagnostic brightness and the inclusion of additional secondary impurities $s$ :

$$
n_{Z_{0}}^{S X R}=\frac{M \cdot \epsilon_{e x p}^{S X R}-n_{e}\left[n_{I} L_{I}^{S X R}+\sum_{s \neq Z_{0}} n_{s} L_{s}^{S X R}\right]}{n_{e} L_{Z_{0}}^{S X R}}
$$

where $M$ is a constant, $\epsilon_{\text {exp }}^{S X R}$ the experimental SXR emissivity, $n$ the density of electrons $(e)$, main ion ( $I$, i.e. either $\mathrm{H}, \mathrm{D}$, or T) and impurities $(s), L^{S X R}$ the SXR-filtered cooling function, i.e. the radiation loss parameter per unit electron and ion densities (in units of $W m^{3}$ ) filtered in energy range detected by the diagnostic. Identically to previous studies, the application of equation 2.1 is limited radially by the view of the SXR camera $\mathrm{V}$ used in the analysis and by the cutoff in the SXR filter-function Te $>1.5 \mathrm{keV}$. The ionization balance and cooling functions (total and SXR-filtered) are all modelled in local- 
ionization-equilibrium (LIE), i.e. neglecting the effect of transport. As already shown in previous publications, this is a valid assumption for the core plasma, especially for mid/high-Z impurities, but may be violated in the pedestal region due to large gradients and fast transport timescales in comparison to the ionization and recombination rates. The atomic data used in this work comes from Pütterich et al. $(2012)$; Casson et al. $(2015)$ for the SXR cooling functions and the ionization/recombination, from Pütterich et al. (2019) for the total radiated power.

While the solution of this equation neglecting secondary impurities is straightforward, including even just one increases dramatically the number of free parameters. First of all each impurity typically has its own source term which depends on its origin (main chamber PFCs, ICRH antenna limiters, NBI duct scraper, divertor tiles, gas valves, etc.) and can be independently influenced by other actuators (gas puffing, ELM frequency, heating power, etc.). Moreover, their radial transport depends strongly on mass and charge, especially in JET high power discharges where the central impurity transport is mostly neoclassical (Casson et al. 2015, Angioni et al. 2015, 2014). Finally, heavy elements are often asymmetric on the flux surfaces, driven mainly by centrifugal forces (Odstrcil et al. 2018, Casson et al. 2015, Reinke et al. 2012, Ingesson et al. 1998), while lighter elements are not. Solving equation 2.1 with secondary impurities therefore requires a certain number of assumptions driven by the present theoretical understanding of impurity transport.

\subsection{Inclusion of multiple low-Z impurities}

As in the original method (Sertoli et al. 2018), light elements are included in the analysis assuming that their concentration profile flat and symmetric on the flux surface $n_{Z_{1}}(\rho ; t)=c_{Z_{1}}(t) \cdot n_{e}(\rho ; t)$. Due to their low mass and in ion-temperature-gradient (ITG) turbulence regimes such as those usually encountered at JET, these assumptions are supported by the current theoretical understanding (Angioni et al. 2011; Bonanomi et al. 2018, McDermott et al. 2018, Bourdelle et al. 2018, Kappatou et al.| 2019).

The concentration is free to evolve in time and is calculated from the visible Bremsstrahlung LoS-averaged $Z_{\text {eff }}$ measurement (Behringer et al. 1986) accounting for contributions of all the other impurities to the LoS-integral:

$$
c_{Z_{1}}(t)=\frac{L \cdot\left(Z_{e f f}(t)-1\right)-\sum_{s \neq Z_{1}} \int_{L} \frac{n_{s}(\rho, R ; t)}{n_{e}(\rho ; t)}\left[\left\langle q_{s}(\rho ; t)\right\rangle^{2}-\left\langle q_{s}(\rho ; t)\right\rangle\right] d l}{\int_{L}\left[\left\langle q_{s}(\rho ; t)\right\rangle^{2}-\left\langle q_{s}(\rho ; t)\right\rangle\right] d l}
$$

$n_{s}$ and $n_{e}$ are the impurity and electron densities respectively, $\left\langle q_{s}\right\rangle$ the impurity average charge (in units of the elementary charge), $L$ the LoS length and the sum is performed over all impurities excluding $Z_{1}$. Differently from Sertoli et al. (2018), the contribution from each impurity is thus modelled self-consistently along the LoS accounting for poloidal asymmetries of the mid-/high-Z impurity densities $n_{s}(\rho, R ; t)$. In this and later equations, quantities assumed symmetric on the flux-surface (impurity average charge, electron temperature and density, factors, etc.) will have a radial dependence $(\rho)$, while those which may exhibit polidal asymmetries are estimated in $2 \mathrm{D}$ on the $(R, z)$ plane and will have a radial dependence $(\rho, R)$. The flux-surface label $\rho$ is retained instead of $z$ in order not to lose the information on the parent flux-surface. The flux-surface label $\rho$ used in from now on corresponds to the normalized poloidal flux coordinate $\rho_{\text {pol }}=$ $\sqrt{\left(\psi-\psi_{a}\right) /\left(\psi_{s}-\psi_{a}\right)}$, where indices $s$ and $a$ refer to the separatrix and the magnetic axis respectively. 
A secondary low-Z impurity $Z_{2}$ can be added with the additional constraint that the concentration is constant in time $n_{Z_{2}}(\rho, t)=c_{Z_{2}} \cdot n_{e}(\rho, t)$. For the case of JET-ILW discharges without impurity seeding, Be is used as primary low-Z $\left(Z_{1}\right)$. If instead seeding takes place, Be is set as the constant background $\left(Z_{2}\right)$ and the seeding gas is set as primary and left free to evolve in time. The constant Be concentration is calculated from the $Z_{\text {eff }}$ measurement prior to the seeding phase assuming it is the only other low-Z impurity. Typical concentrations are of the order of $0.5-2.0 \%$.

After having applied equation 2.1 with the chosen low-Z impurities, if the main impurity $Z_{0}$ is tungsten the first guess of the impurity density is re-scaled comparing the result with the independent measurement from VUV spectroscopy (Schwob et al. 1987, Pütterich et al. 2008):

$$
n_{Z_{0}}(\rho, R ; t)=\left\langle c_{W}(t) / c_{W}^{S X R}(t)\right\rangle_{t} \cdot n_{Z_{0}}^{S X} R(\rho, R ; t)
$$

where $c_{W}$ is the VUV spectrometer measurement and $c_{W}^{S X R}$ the simulated one integrating $n_{W}^{S X R}$ from eq. 2.1 along the LoS of the VUV spectrometer and weighing it on the fractional abundance envelope of the measured ionization stages. This provides a first consistency check of the absolute $\mathrm{W}$ density with the assumption that it is the major contributor to the SXR emissivity, but accounting for the fact that contributions from other impurities might be present. For further details, see Sec. II A of Sertoli et al. (2017) and references therein.

This density is then used to evaluate a re-calibration factor for the SXR emissivity:

$$
\begin{aligned}
M & =\left\langle\frac{\epsilon^{S X R}(\rho, R ; t)}{\epsilon_{\text {exp }}^{S X R}(\rho, R ; t)}\right\rangle \\
& =\left\langle\frac{n_{e}(\rho ; t)\left[n_{I}(\rho ; t) L_{I}^{S X R}(\rho ; t)+\sum_{s} n_{s}(\rho, R ; t) L_{s}^{S X R}(\rho ; t)\right]}{\epsilon_{\exp }^{S X R}(\rho, R ; t)}\right\rangle_{(\rho, R ; t)}
\end{aligned}
$$

where $\epsilon_{\exp }^{S X R}$ is the result of the deconvolution routine and the average is performed over the whole time-range of interest and over the plasma radius within the range of validity of the analysis, and contributions of all other secondary impurities $s$ are accounted for. The process is iterated a few times until convergence (two iterations have been found to suffice) after which the re-scaling vs. the W concentration measurement is abandoned and the SXR multiplication factor is the only correction that is retained. If the main impurity is not $\mathrm{W}$, then this multiplication factor is set to $M=3$, value previously estimated by comparing the SXR emissivity with bolometry in plasmas with dominating low-Z impurity and negligible contributions from mid-/high-Z elements (Pütterich et al. 2012). As a confirmation to this previous estimate, multiplication factors of the order of $2.5-3.5$ have been found for all those cases that show good consistency with other diagnostic measurements, where the main impurity is $\mathrm{W}$ and the $\mathrm{W}$ concentration measurements from VUV was available. This re-calibration factor $M$ is JET-specific and its origin is still not understood. It could be tied with uncertainties in the Be-filter thickness, in the atomic data or in the diagnostic acquisition, but the fact that it is constant over time and independent of plasma scenario legitimizes treating it as a re-calibration factor.

After having calculated the multiplication factor, equation 2.1 is re-applied and the whole procedure repeated until convergence (two more times are typically enough). Consistency checks with independent measurements are then applied to falsify or confirm the results obtained so-far. 


\subsection{Consistency-checks versus independent measurements}

On top of the consistency checks already used in the original method (Sertoli et al. 2018) which are listed below as (a-c), a few more have been implemented to provide further robustness to the methodology:

(a) if the main impurity $Z_{0}$ is tungsten, compare its time evolution with the independent concentration measurement from VUV spectroscopy.

(b) from the observed LFS-HFS asymmetry of $Z_{0}$ evaluate the expected toroidal rotation and compare with CXRS measurements.

(c) including contributions from all ions, calculate the total radiation (in 2D) and compare with estimates tomographic reconstructions of the bolometry diagnostic.

(d) integrate the estimated total radiation along the LoS of the bolometry diagnostic and compare with the experimental values.

(e) if a seeding gas is included, compare the time evolution and absolute value of the independent concentration measurement from CXRS.

While checks (a-c) and (e) are relatively straightforward, comparing with LoS integrals of bolometry requires an extrapolation of the results beyond the range of validity of the analysis. This is a delicate part and will be explained in the next section.

\subsection{Inclusion of a secondary mid-/high-Z impurities}

If the solution of equation 2.1 including only low-Z impurities yields estimate that do not match the consistency checks within the estimated experimental uncertainties, one more impurity $Z_{3}$ can be added to the mix. This can be a mid-/high-Z element and its time evolution, profile shape and asymmetry is modelled on the basis of $n_{Z_{0}}$ with the following assumptions:

(i) both impurities $Z_{0}$ and $Z_{3}$ are at equilibrium, the impurity sources and sinks don't change during the time-range of analysis and the time-averaging is long enough (typically $20 \mathrm{~ms}$ ) such that the net particle flux through the separatrix is zero. If this is not the case, the sources and sinks of both impurities are assumed to behave identically.

(ii) the central peaking of $Z_{0}$ and $Z_{3}$ is driven by neoclassical processes.

(iii) poloidal asymmetries are driven mainly by centrifugal effects.

If the peaking of the impurity density profiles in the plasma centre is neoclassically driven, as it typically is in the centre of JET H-mode plasmas (Angioni et al. 2015, Casson et al. 2015), the radial impurity flux can be modelled following Breton et al. (2018b, eq. 7). If the particle flux is zero and the profiles are in equilibrium, the normalized impurity density gradient can then be calculated as:

$$
\left\langle\frac{R}{L_{n_{s}}}\right\rangle_{F S A} \propto q_{s}\left[\frac{R}{L_{n_{I}}}+\frac{R}{L_{T_{I}}}\left(\frac{H}{K}+\frac{H_{0} f_{c}}{K} \frac{P_{B}}{P_{A}}\right)\right]
$$

where $R$ is the tokamak major radius, $L_{A}=A / \nabla A$ the gradient length of quantity $A$, $m_{I}$ the mass of the main ion $I$ and $T_{I}$ its temperature, $q_{s}$ the impurity charge, $H, H_{0}$ and $K$ factors that depend on the collisionality, $f_{c}$ the fraction of circulating particles, $P_{A}$ and $P_{B}$ the geometrical factors related to poloidal asymmetries (Casson et al. 2015; Breton et al. 2018b). All quantities are flux-surface-averages (FSA), including the left hand side of the equation. The subscript $F S A$ will be neglected from now on, but the symbol \langle\rangle will retain the meaning of flux-surface-averaged quantity.

For peaked profiles, the pinch term proportional to $R / L_{n_{I}}$ will dominate over both the ion temperature screening term proportional to $R / L_{T_{I}}$ as well as turbulence. Neglecting 
at first order the temperature screening term, the normalized impurity density gradient becomes directly proportional to the main ion gradient multiplied by the impurity charge $q_{s}$. Using the previously estimated density profile of impurity $Z_{0}$, the gradients of the secondary impurity $Z_{3}$ can be calculated as:

$$
\left\langle\frac{R}{L_{n_{Z_{3}}}}\right\rangle \approx \frac{q_{Z_{3}}}{q_{Z_{0}}}\left\langle\frac{R}{L_{n_{Z_{0}}}}\right\rangle
$$

Inverting this equation will then yield the shape of the impurity density profile:

$$
\left\langle n_{Z_{3}}\right\rangle=\exp \left[\int_{\rho}\left\langle\frac{1}{L_{n_{Z_{0}}}}\right\rangle \frac{q_{Z_{3}}}{q_{Z_{0}}} d r\right]
$$

Since this re-adaptation of the flux-surface-averaged impurity density gradient is not performed in absolute terms with respect to $R / L_{n_{I}}$, but relative to the primary impurity $Z_{0}$ whose gradient is determined experimentally, second-order effects mitigating the impurity peaking coming from neoclassical temperature screening and turbulence are still somewhat retained.

In regions of the plasma where the profile if impurity $Z_{0}$ is flat or hollow, then neoclassical temperature screening or turbulence will be dominating of over the neoclassical pinch term. In JET-ILW plasmas the former is usually responsible for effects close to the plasma centre, while the latter is typically the dominating transport mechanism for radii $\rho>0.3-0.4$ and will lead to profiles $n_{s} \propto n_{e}$ (Casson et al. 2015, Angioni et al. 2015, Bourdelle et al. 2018). In this case, the flux-surface-averaged impurity density gradient of $Z_{3}$ will be set equal to that of the primary high-Z impurity $L_{n_{Z_{3}}}=L_{n_{Z_{0}}}$.

For the determination of the absolute value and time evolution, assumption $(i)$ provides the necessary framework by imposing that the flux-surface-averaged density profile of $Z_{3}$ follows closely that of $Z_{0}$. The result of equation 2.7 is thus normalized setting the total number of $Z_{3}$ ions in the confined region equal to that of $Z_{0}$ at each time-point:

$$
\left\langle n_{Z_{3}}\right\rangle=\left\langle n_{Z_{3}}\right\rangle \frac{\int\left\langle n_{Z_{0}}\right\rangle d V}{\int\left\langle n_{Z_{3}}\right\rangle d V}
$$

and finally multiplied by a constant $C$ which is a single number for the whole time-range of analysis:

$$
\left\langle n_{Z_{3}}\right\rangle=C \cdot\left\langle n_{Z_{3}}\right\rangle
$$

This constant has the meaning of the fraction of total impurity density of $Z_{3}$ with respect to $Z_{0}$. This is evaluated applying consistency check (d) comparing the LoS integrals of the bolometric diagnostic with its synthetic value estimated from the $2 \mathrm{D}$ maps of the total radiation calculated including contributions from the main ion and all impurities:

$$
\frac{1}{C}=\left\langle\frac{1}{N} \sum_{k=1}^{N} \frac{\int_{L_{k}}\left[n_{e}(\rho ; t)\left(n_{I}(\rho ; t) L_{I}^{\text {tot }}(\rho ; t)+\sum_{s} n_{s}(\rho, R ; t) L_{s}^{t o t}(\rho ; t)\right)\right] d l}{B_{k}^{\text {bolo }}(t)}\right\rangle_{t}
$$

where the sum is performed over $N$ lines-of-sight of lenght $L_{k}$ and $B_{k}^{\text {bolo }}$ are the experimental LoS-integrals. Since the method for equation can be applied above $T_{e}\left(\rho_{\max }\right)=$ $1.5 \mathrm{keV}$, the densities $n_{Z_{0}}$ and $n_{Z_{3}}$ must be extrapolated from $\rho_{\max }$ up to the separatrix. This can be done in a number of ways, including scaling the electron density over the whole region $\rho>\rho_{\max }$ or extrapolating the value or the derivative up to a specified 
radius $\rho_{\text {interp }}$ and then following the shape of the electron density scaled to match the impurity density at $\rho_{\text {interp }}$. All of these methods are performed on the midplane values of the 2D impurity densities, independently for LFS and HFS, to maintain the observed asymmetry at $\rho_{\max }$. For the pedestal region, in order to artificially compensate for the underestimated cooling functions calculated neglecting transport, the impurity density is slightly enhanced by increasing the pedestal value to $n_{Z_{0}}(\rho>0.9)=\left(n_{Z_{0}, n_{e}}+n_{Z_{0}}(\rho>\right.$ $0.9)) / 2$..

The last point to clarify is how to evaluate the poloidal asymmetry of $Z_{3}$. This is straightforward if assumption (iii) is valid and an estimate of the toroidal rotation profile is available. Following eq. (9) of Odstrcil et al. (2018) and neglecting the asymmetries driven by fast particles (first term on the right-hand-side), the impurity density on a flux surface $\rho$ at a generic mayor radius $R$ can be calculated with respect to the value at a reference major radius $R_{0}$ :

$$
n_{s}(\rho, R ; t) \approx n_{s}\left(\rho, R_{0} ; t\right) \exp \left[\lambda_{s}(\rho ; t)\left(R(\rho ; t)^{2}-R_{0}(\rho ; t)^{2}\right)\right]
$$

where the asymmetry parameter $\lambda_{s}$ is fully determined by knowing the main ion and impurity masses $\left(m_{I}\right.$ and $\left.m_{s}\right)$, the average impurity charge $\left\langle q_{s}\right\rangle$, the effective charge $Z_{e f f}$, the electron and ion temperatures $T_{e, I}$, and the toroidal rotation frequency $\omega_{\phi}$ :

$$
\lambda_{s}(\rho ; t) \approx \frac{m_{s} \omega_{\phi}(\rho ; t)^{2}}{2 T_{I}(\rho ; t)}\left(1-\frac{\left\langle q_{s}(\rho ; t)\right\rangle}{m_{s}} \frac{m_{I} Z_{e f f}(\rho ; t) T_{e}(\rho ; t)}{T_{I}(\rho ; t)+Z_{e f f}(\rho ; t) T_{e}(\rho ; t)}\right)
$$

The impurity density at the chosen reference radius $R_{0}$ on the low-field-side (LFS) midplane can then be estimated by inverting eq. 2.11 and calculating the flux-surfaceaveraged value of the exponent:

$$
n_{s}\left(\rho, R_{0} ; t\right)=\frac{n_{s}(\rho ; t)}{\left\langle\exp \left[\lambda_{s}(\rho ; t)\left(R(\rho ; t)^{2}-R_{0}(\rho ; t)^{2}\right)\right]\right\rangle}
$$

All other quantities in equation 2.12 are already included in the analysis or selfconsistently calculated using the available profile data.

The toroidal rotation and ion temperature are taken from CXRS measurements on impurity ions (e.g. $N e X$ ) and are assumed equal for all impurities. This assumption is consistent with previous findings (Baylor et al. 2004, Lebschy 2018; Grierson et al. 2019) and can be confirmed here for consistency using the radial force balance equation $E_{r}=\nabla p / q n+v_{\phi} B_{\vartheta}-v_{\vartheta} B_{\phi}$. Since the radial electric field will be equal for all species, with the assumption that their temperatures are the same, that their peaking follows equation 2.6 and that the poloidal flow term is negligible at first order, the difference between the toroidal velocities of two ions will be:

$$
v_{\phi, s_{1}}-v_{\phi, s_{2}} \approx-\frac{\nabla T}{B_{\vartheta}}\left(\frac{q_{s_{2}}-q_{s_{1}}}{q_{s_{2}} q_{s_{1}}}\right)
$$

with $\nabla T$ given in $(e V / m)$ and $B_{\vartheta}(T)$. This leads to small differences for impurity ions, and slightly larger values when comparing impurities and main ion rotation. For the example discharge shown in the next section, the toroidal rotation difference between main ion and the measured neon is expected to be of the order of a few tens of $\mathrm{km} / \mathrm{s}$, while the difference between tungsten or nickel turns out to be a only a few $\mathrm{km} / \mathrm{s}$, i.e. less than a percent of the measured toroidal rotation and therefore negligible. 

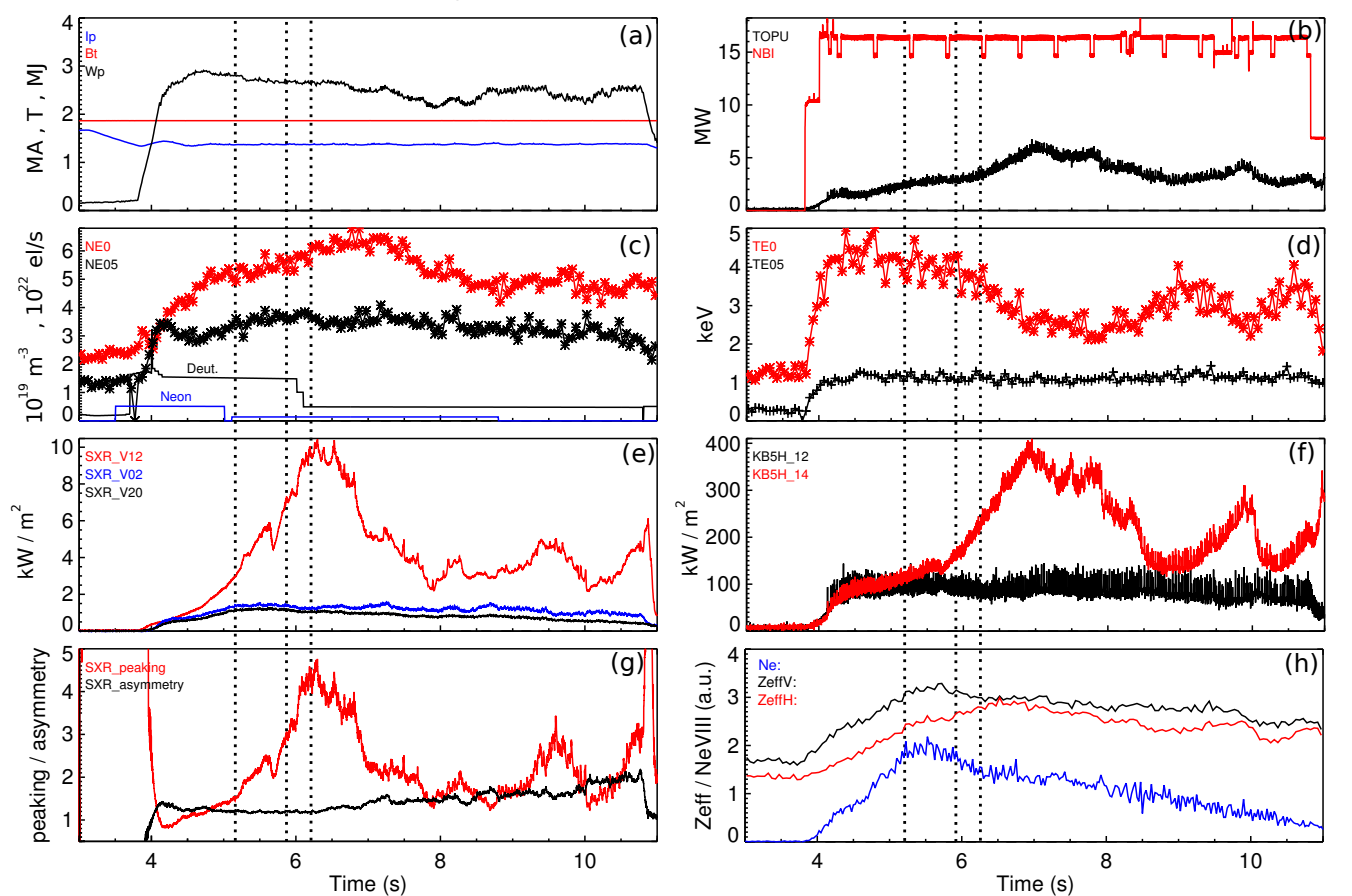

Figure 2. Time evolution of the main plasma parameters of 90279: (a) plasma current $(M A)$, toroidal magnetic field $(T)$ and plasma stored energy $(M J)$; (b) NBI heating power $(M W)$ and total bulk radiation $(M W)$ estimated from the vertical bolometer camera; (c) electron density at the centre (red) and at mid-radius (black) $\left(10^{19} \mathrm{~m}^{-3}\right)$, deuterium and neon total fueling rate $\left(10^{22} \mathrm{el} / \mathrm{s}\right)$; (d) electron temperature at the centre (red) and mid-radius (black); (e) SXR LoS-integral crossing the plasma centre (red) and with impact parameter $\rho_{\text {min }} \approx 0.5$ viewing the LFS (blue) and HFS (black); (f) bolometer LoS-integral viewing the centre (red) and with $\rho_{\text {min }} \approx 0.4$ (black); (g) SXR peaking (red) and asymmetry (black) factors; (h) $Z_{\text {eff }}$ measurements along the vertical (black) and horizontal (red) LoS and intensity of the 780.32 (A) spectral line of NeVIII measured by the VUV spectrometer KT2.

\section{Example discharge}

The discharge chosen to explain the method is a neon-seeded hybrid discharge 90279. This is a $1.4 M A, 1.8 T$ pulse, with $16 M W$ NBI heating and Ne seeding (blue trace in fig. 2b) starting before the main NBI heating is ramped-up (red trace in fig. $2 \mathrm{~b}$ ). It exhibits slow but steady electron density peaking in the first half of the flat-top (fig. 22), accompanied by strong impurity peaking (fig. $2 \mathrm{p}-\mathrm{g}$ ) and erosion of the central electron temperature (fig. 2 $\mathrm{d}$ ). The $Z_{\text {eff }}$ increases strongly in these first seconds (fig. 2h), the total radiated power from the bulk plasma increases slightly (black in fig. $2 \mathrm{p}$ ) and the plasma stored energy is slightly eroded (fig. 2a), but the discharge nonetheless survives up to the end of the programmed flat-top. This discharge exhibits fishbone activity in time-range $\sim[44.7,45.3](s)$, then saturated $1 / 1$ modes until $46.2(s)$, but no sawtooth cycling, and a $3 / 2$ mode appearing at $7.3(s)$. The absence of mode $1 / 1$ activity later in the pulse is attributed to modifications in the current density profile with $q(0)>1$ as a consequence of the hollowing of the electron temperature profiles driven by impurity accumulation (Pucella et al. 2019).

The first two seconds of the flat-top $(4.5-6.5)$ during impurity accumulation are of interest here for the purpose of explaining the analysis methodologies. Three timepoints have been chosen along this accumulation path: $t=5.17,5.85,6.17 \mathrm{~s}$ (vertical 

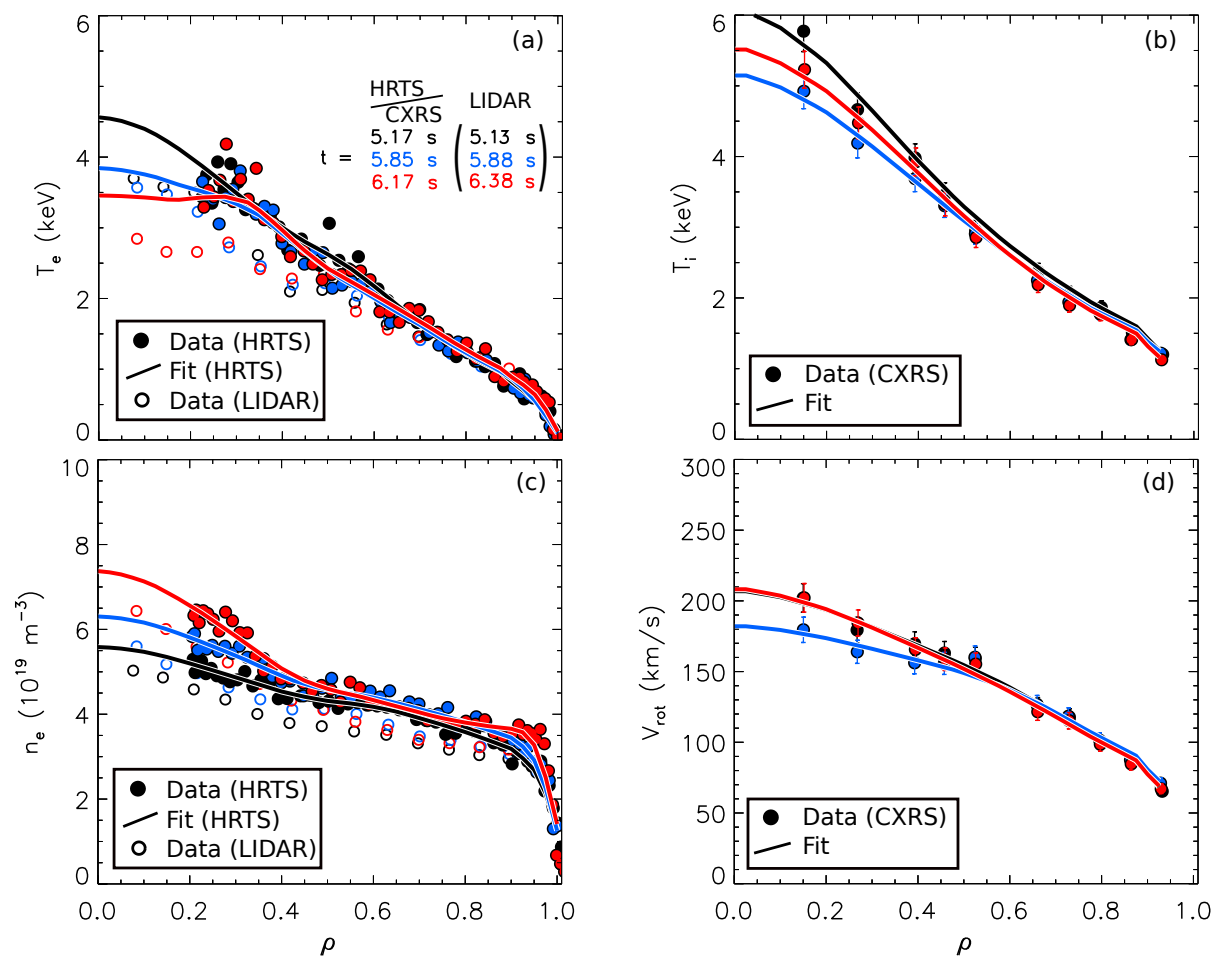

Figure 3. Data (points) and fits (continuous lines) of electron (a) and ion (b) temperature, electron density (c) and toroidal rotation (d) for the three time-points labeled in (a), shown in figure 2 as dashed vertical lines.

dashed lines in fig. 2p. All quantities used in the analysis are mapped on the EFIT equilibrium reconstruction accounting for a $3 \mathrm{~cm}$ shift necessary to match the HRTS electron temperature at the separatrix to $100 \mathrm{eV}$. This shift is applied not only to HRTS, but to all diagnostic LoS and measurement positions.

Ion temperature and toroidal rotation are taken from CXRS measurements of $\mathrm{NeX}$ $5249(\AA)$, with a time resolution of $50(\mathrm{~ms})$ and covering the whole radial range (fig. $3 \mathrm{~b}$, d). The HRTS diagnostic is used for electron density and temperature profiles, with a time resolution of $50(\mathrm{~ms})$. It's radial profile does not reach plasma centre (fig. 1), but the density peaking and the decrease in central temperature for the later time-point are still detected (fig. 3p). This is confirmed when comparing with LIDAR Thomson scattering data which measures up to the plasma centre but at a low time resolution of $250(\mathrm{~ms})$ (circles in fig. 3c). The differences between HRTS and LIDAR data are first of all due to different measurement times of the two diagnostics (label in fig. 3 a). Especially for the last time-point considered (6.17 $s$, shown in red) when the impurity accumulation has progressed increasing the central radiation (red in fig. $2 \mathrm{f}$ ) and reducing further the central electron temperature (red in fig. 2d). The remaining difference, with LIDAR values approximately $10 \%$ lower across the whole radial range, is instead probably due to uncertainties in the equilibrium reconstruction. When performed without internal constraints, EFIT is known to overestimate the Shafranov shift which will affect LIDAR more than HRTS since it measures closer to the midplane (green diamonds in fig. 1). The effect of these uncertainties on the final result is anyway minimal because the error on the electron profile fits is $\sim 6 \%$, i.e. roughly of the same order of magnitude.

The 2D SXR emissivity maps resulting from the inversion routine for these three time- 



FIgURE 4. SXR emissivity profiles at the chosen time-points (see label): (a-c) 2D SXR emissivity (identical logarithmic color scale); (d) back LoS-integrals of the 2D emissivity compared with the experimental data (continuous lines and points with error bars, respectively) plotted vs. LoS impact parameter; (e) cut of the 2D SXR emissivity at midplane, positive $\rho$ corresponding to LFS, negative to HFS.

points are shown in figure 4 a-c. The back LoS-integral of these poloidal maps compare well with the experimental data for all $28 \mathrm{LoS}$ of SXR camera V at all three time-points considered (fig. 4d). A cut at the midplane (fig. 4e, positive $\rho$ corresponding to LFS, negative to HFS) reveals an order of magnitude increase in central emissivity and a slight LFS asymmetry very similar for all time-points. For the estimation and propagation of the experimental uncertainties from the SXR LoS-integrals to the inverted emissivity profiles, from the electron temperature to the cooling factors and the final propagation to the resulting high-Z impurity density, the methodologies described in-depth in sections IIB and II of Sertoli et al. (2018) have been applied.

Tungsten is used as primary high-Z impurity $\left(Z_{0}\right)$ and two low-Z impurities are included from the start: beryllium as constant background $\left(Z_{2}\right)$ and the seeded neon left free to evolve in time $\left(Z_{1}\right)$. The Be concentration has been set to $2.0 \%$ to match the $Z_{\text {eff }}$ value at $4 s$ before the increase in effective charge takes place (2d). The horizontal $Z_{\text {eff }}$ measurement $Z E F H$ has been chosen because it is thought to be less affected by reflections on PFCs that can lead to an artificial increase of the measured 
value. As a further consistency check, the analysis has also been performed using the vertical LoS measurement $Z E F V$ and the results will be reported in section 3.7. The statistical uncertainties on these measurements are estimated to be $<5 \%$, including the propagation of electron density and temperature uncertainties used for the calculation, so the difference between the two measurements are outside of the confidence interval.

With a step-wise approach, including more impurities in the mix, checking the consistency between diagnostics and trying to falsify assumptions or testing the trustworthiness of different measurements, four cases have been explored:

1. W as primary $\left(Z_{0}\right)$, re-normalized to the VUV concentration measurement $c_{W, q c}$;

2. step 1. \& W density scaled to match the most central bolometer LoSs;

3. step 1 . \& $\mathrm{Ni}$ as secondary mid-Z $\left(Z_{3}\right)$ scaled to match bolometer measurements;

4. Ni as primary $\left(Z_{0}\right)$, SXR multiplication factor $M=3.0$;

Case 1. represents the standard analysis, where the $\mathrm{W}$ density is assumed to be the only high-Z impurity in the plasma and the VUV measurement of the W concentration is used as cross-calibration for the initial results. In case 2., the VUV measurement is deemed untrustworthy and the $\mathrm{W}$ density profile is further re-scaled so that the total radiation matches what observed along the most central LoSs of both bolometer cameras $\mathrm{H}$ and $\mathrm{V}$. The extrapolation of the $\mathrm{W}$ density beyond the range of validity of the analysis $\left(\rho_{\max } \sim 0.6\right)$ is performed for all cases following the shape of the electron density profile.

For case 3 ., if $\mathrm{W}$ is not the only mid-/high-Z impurity in the plasma and the VUV measurement is trustworthy, nickel is included in the mix and its absolute value scaled so that the simulated total radiated power including all ions matches the bolometry LoS integrated measurements. Nickel, iron as well as copper are known to be present in JET plasmas and are routinely identified by VUV survey spectrometers. The former have been seen to correlate with the use of the ICRH antennas (Czarnecka et al. 2011), while copper with NBI heating, its influx caused by the interaction of fast NBI neutrals with the NBI duct-scraper. Due to the very similar mass and charge of these elements, nickel is used as a proxy for mid-Z impurities owing to the availability of atomic data and cooling functions.

Finally, case 4. turns the problem upside-down exploring what happens if $\mathrm{W}$ has been erroneously chosen as primary impurity and $\mathrm{Ni}$ is the main radiator. In order to better understand the consequences of the above assumptions and visualize the main differences on the results and consistency-checks, the first three cases will be discussed and shown in parallel (figures 5 and 6 ). The fourth will instead be shown at the end of this section as final confirmation that case 3 . is the best solution.

\subsection{Effects on Zeff}

The different assumptions for the impurity content lead to noticeable changes in the contribution to the $Z_{\text {eff }}$ LoS integrated measurement (fig. 5 a). The low-Z impurities $(\mathrm{Be}+\mathrm{Ne})$ are in general the major contributors (short dashes). Tungsten contributes little because of its low concentration (continuous lines in fig. 5k), reaching a maximum of $\Delta Z_{\text {eff }} \sim 0.2$ if its density is re-scaled to fill in for the missing radiated power (case 2.). When nickel is included (case 3.), it becomes a major contributor towards the end of the accumulation process, accounting for approximately half of the measured LoSintegrated value. The neon concentration (continuous red line in fig. 5b) is therefore lowest in case 3 . (about a factor 2), having to account for less $Z_{\text {eff }}$ than in the other two cases. When not including nickel, the match of the estimated neon concentration with the radially averaged CXRS data (dashed line) is way outside the confidence interval 


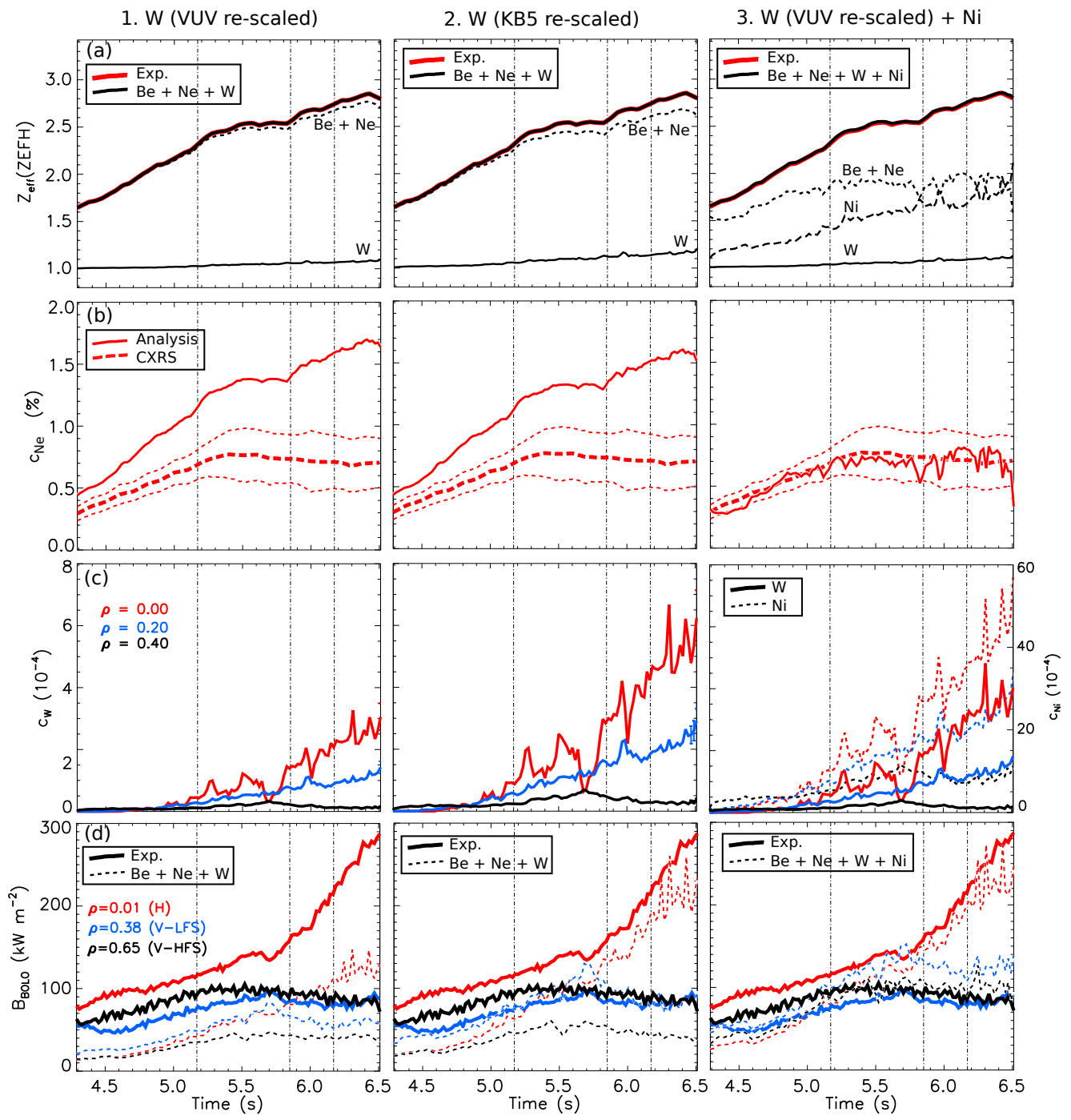

Figure 5. Time evolution of various quantities for cases 1.-3.: (a) experimental $Z_{\text {eff }}$ measurement $Z E F H$ (red) compared with the back LoS-integrated value (thick black line) and single contributions from $B e+N e, W$ and $N i$ (only for case 3.); (b) radially averaged experimental Ne concentration from CXRS (dashed line with confidence interval) compared with the estimated value (continuous line); (c) flux-surface-averaged W concentration at three different radial positions (colour coded as in label), and of the Ni density (case 3. only, dashed lines, axis of reference on the right); (d) experimental bolometric LoS-integrated measurements (continuous lines) from three $\operatorname{LoS}(\mathrm{V}=$ vertical, $\mathrm{H}=$ horizontal camera, $\rho=$ impact parameter) compared with the back LoS-integrated values (dashed lines).

of the CXRS measurement which has been estimated propagating a $15 \%$ error with the standard deviation of all radial points. With the contribution of nickel (case 3.), the match is instead extremely good for the whole time-range of analysis.

\subsection{Effects on total radiation}

The flux-surface-averaged $\mathrm{W}$ concentration (continuous lines in fig. 5p) reaches a maximum of $\sim 3-4 \cdot 10^{-4}$ in the centre (red) when normalized to the VUV concentration 
1. W (VUV re-scaled)
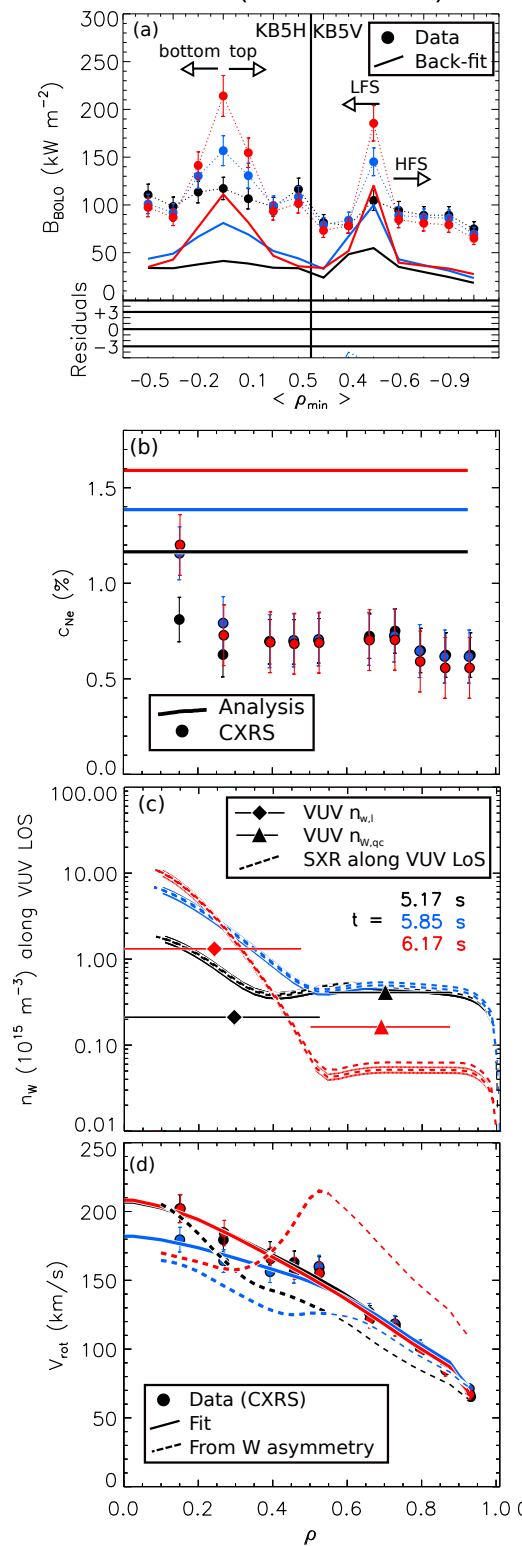

2. W (KB5 re-scaled)
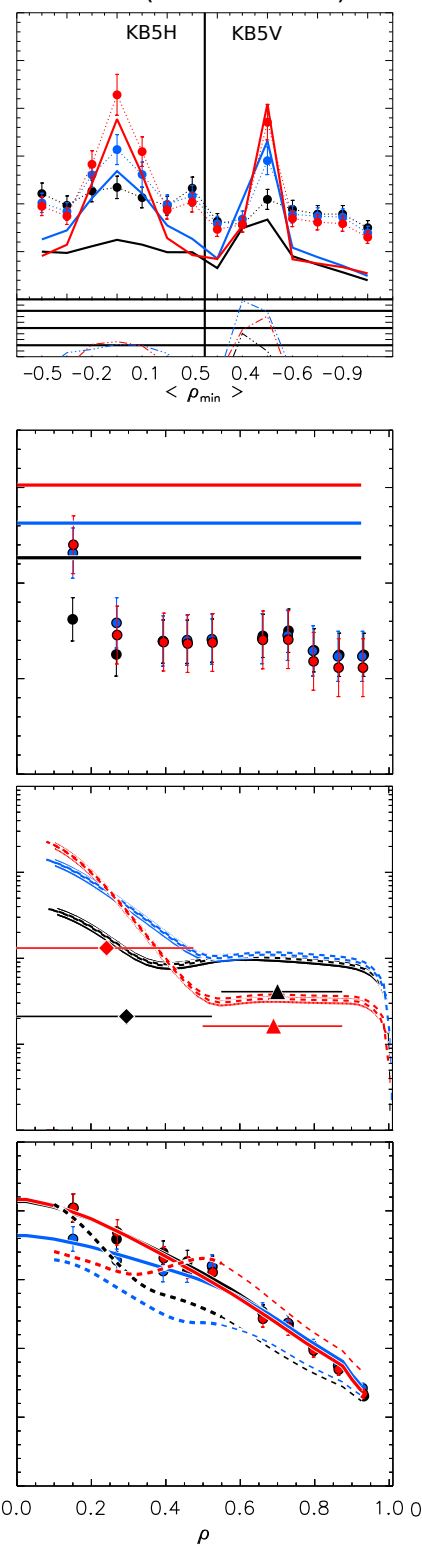

3. W (VUV re-scaled) $+\mathrm{Ni}$
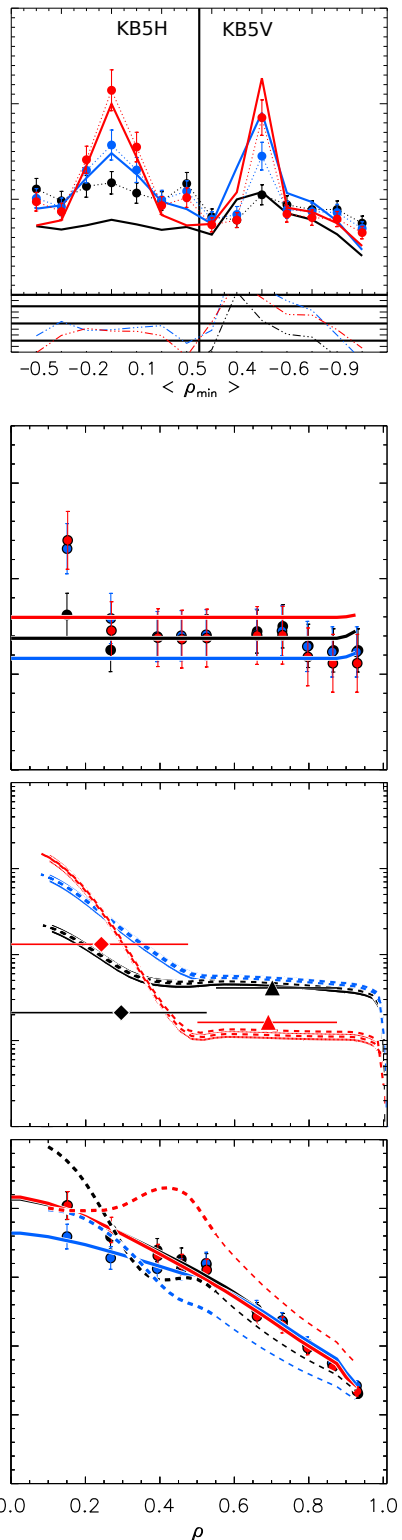

FiguRE 6. Profile consistency checks for cases 1.-3.: (a) experimental bolometric LoS-integrated measurements (points with error bars) for all LoS shown in fig. 1 compared with the back LoS-integrated values (continuous line); ; (b) profile of the experimental Ne concentration measurement from CXRS (points) compared with the estimated value (continuous line); (c) W density measured by the VUV spectrometer (diamonds and triangles) compared to the estimated value along the spectrometer LoS (dashed line, coloured band); toroidal rotation measurements from CXRS (points) and its fits (continuous lines) compared to the value estimated from the $\mathrm{W}$ density asymmetry (dashed line). 
measurement (cases 1. and 3.) changing slightly if nickel is included in the mix (case 3.). Double that amount is needed to improve the match with bolometric measurements (fig. 5d) as in case 2. It is clear from these latter plots that the match of the back LoS-integrals (dashed lines) with the experimental data for three LoS (colour-coded) over the whole time-range of interested is best for case 3. The total amount of Ni needed to match the bolometer signals is approximately one order of magnitude larger than that of $\mathrm{W}$ (dashed lines, case 3. fig. 5. .

The consistency check with bolometry is further enhanced comparing all available LoS not viewing the divertor (fig. 6a). This comparison clearly shows that increasing the $\mathrm{W}$ density to match the central LoSs does not provide a satisfactory match neither for horizontal LoSs viewing the top and bottom of the plasma, nor for the vertical LoS viewing the HFS. The situation improves considerably when including nickel, with an almost perfect match for all LoS of the vertical camera and a very good agreement for the horizontal camera. The reasons for are that nickel exhibits a lower peaking factor and lower centrifugal asymmetry due to its lower mass and charge (equations 2.6 and 2.11). This can be seen in the plots of the $\mathrm{W}$ and Ni density profiles at midplane at the timepoints of interest for case 3. (fig. 7 a,d). Including also effects from the shape of the cooling factor, with nickel radiating less at higher electron temperatures than tungsten, a global reduction in radiation peaking and an increased radiation on the HFS will take place, improving the match with the bolometer LoSs viewing away from the plasma centre.

\subsection{Match with CXRS Ne concentration}

The comparison of the estimated Ne concentration with the CXRS profiles (fig. 6b) confirms the good match already seen in the time-evolution, but at the same time reveals a peaking in the centre not included in the analysis because of the flat concentration constraint. This experimental point could be an outlier and two independent considerations corroborate this opinion.

The error estimation of the CXRS rotation and temperature measurements relies only on the fit of the spectral lines, while the estimation of the impurity concentration requires knowledge of the beam characteristics including the beam attenuation whose uncertainty is very large close to the plasma centre (Giroud et al. 2008). Moreover, the most central CXRS radial measurement at $\rho \sim 0.05$ (not shown here) has be independently excluded from the analysis due to extremely noisy ion temperature and toroidal rotation result. This means that the beam attenuation in this region is already very large and errors in its estimation could lead to large changes in the calculated impurity density.

The second point is that the experimental peaking is much larger that what expected when calculating the Ne peaking using equation 2.6. Normalizing the profiles to mid radius, the expected central value should be $c_{N e}(\rho=0.15) \sim 0.76,0.75$ and 0.77 for the three time-points respectively. While the difference for the first time-point (black) is within error bars, for the latter two the experimental values are well beyond the $2 \sigma$ boundary.

\subsection{Match with $V U V$}

Calculating the expected W concentration measurement from VUV spectroscopy and comparing it with the experimental values (figure 6c), the best match with the quasicontinuum measurement $c_{W, q c}$ (triangles) is obtained only when accounting for nickel contributions (case 3.). The reason for this is that the presence of nickel accounts for part of the SXR emissivity that was initially ascribed to $\mathrm{W}$, changing the shape of the final $\mathrm{W}$ density profile especially in the later phase of the analysed time-range. The match 

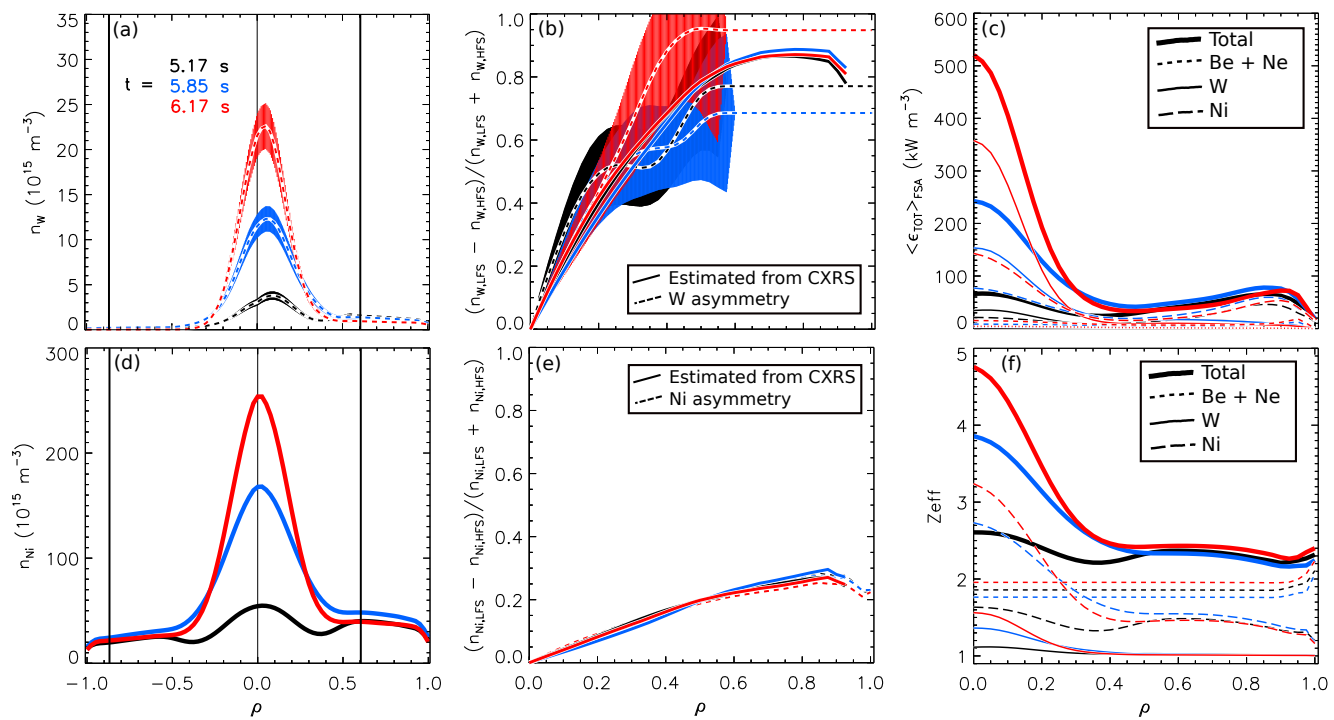

FiguRe 7. Final profile plots for case 3. including nickel: (a) W density at midplane (positive $\rho$ corresponding to LFS, negative to HFS); (b) W density asymmetry (dashed line with confidence band) compared with the estimated value using CXRS measured toroidal rotation; (c) flux-surface-averaged total radiated power and contributions from the different impurities (see label); (d) Ni density at midplane; (e) Ni density asymmetry (dashed line with confidence band) compared with the estimated value using CXRS measured toroidal rotation; (f) $Z_{\text {eff }}$ profile, and contributions from the various impurities (see label).

with the spectral-lines measurement $c_{W, l}$ is instead not optimal for either of the three cases. This is most probably caused by uncertainties in the equilibrium reconstruction: errors in the Shafranov shift will have a large impact on this measurement, but will be more forgiving on the quasi-continuum value which measures further out.

\subsection{Match with toroidal rotation}

The final consistency check is between the measured CXRS toroidal rotation of $\mathrm{NeX}$ and the value estimated from the $\mathrm{W}$ density asymmetry modelled inverting equation 2.11 to extract $\omega_{\phi}$. This match is relatively good for all cases (fig. 6) and this consistency check alone would not be sufficient to distinguish between the three. This is apparent when considering the uncertainties in the $\mathrm{W}$ density asymmetry and viewing the problem in the opposite direction comparing the measured W density asymmetry with the expected one estimated using the CXRS toroidal rotation measurements (figure $7 \mathrm{~b}$ ). It is clear from this plot that the slight differences in asymmetry are all within the confidence interval and therefore cannot be sufficiently discriminated. The errors have been estimated propagating the error of the $\mathrm{W}$ density, calculated by propagating the uncertainties of the deconvoluted SXR emissivity (5\% error from the raw SXR data and deviation of the back-fit with respect to the data) and those of the cooling factors (estimated from upper and lower estimates of the electron temperature given the diagnostic uncertainties and the standard deviation of data points if time-averaging of the raw data is performed). This is clearly a lower estimate, since it does not include uncertainties in either the W atomic data or that of other elements included in the analysis. 

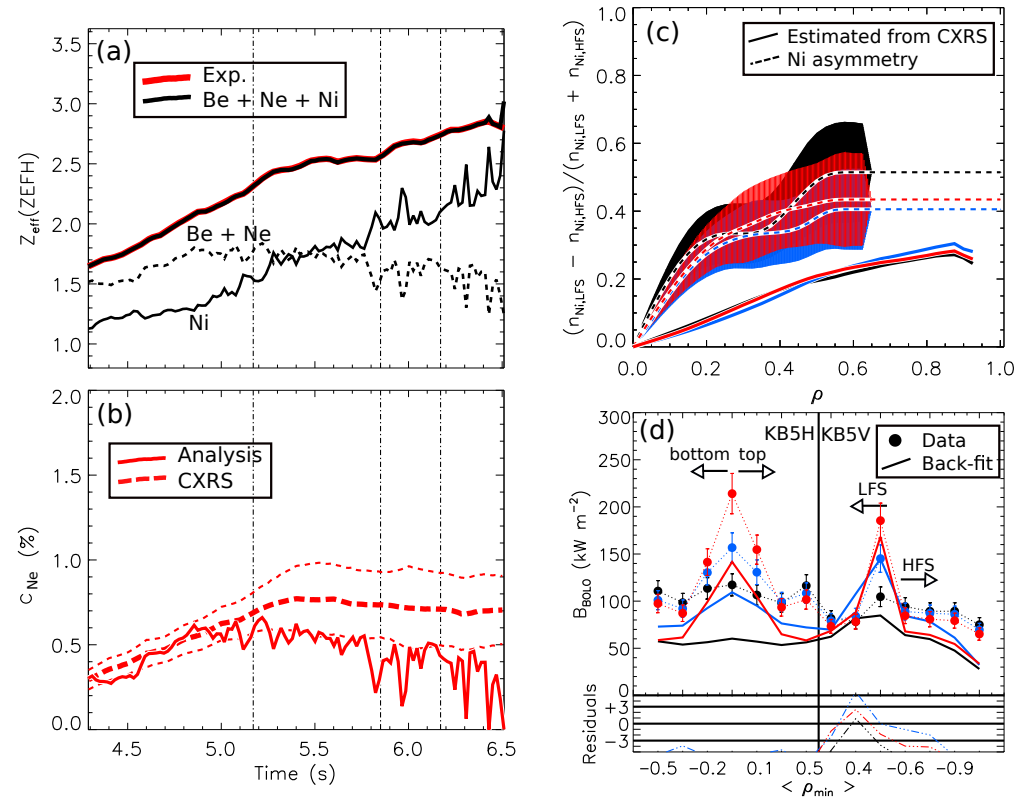

Figure 8. Time-evolution and profile consistency for case 4. (Ni only): (a) experimental $Z_{\text {eff }}$ measurement $Z E F H$ (red) compared with the back LoS-integrated value (thick black line) and single contributions from $\mathrm{Be}+\mathrm{Ne}$ and $\mathrm{Ni}$; (b) radially averaged experimental $\mathrm{Ne}$ concentration from CXRS (dashed line with confidence interval) compared with the estimated value (continuous line); (c) Ni density asymmetry (dashed line with confidence band) compared with the estimated value using CXRS measured toroidal rotation; (d) experimental bolometric LoS-integrated measurements (points with error bars) for all LoS shown in fig. 1 1 compared with the back LoS-integrated values (continuous line).

\subsection{Nickel as main high-Z impurity}

As a final confirmation that including nickel in the impurity mix provides the best match to all measurements but that the presence of $\mathrm{W}$ is of utmost importance and cannot be neglected, figure 8 shows what happens if nickel is the only mid-/high-Z impurity $Z_{0}$. Accounting for most of the SXR emission, the Ni contribution to $Z_{\text {eff }}$ dominates the measurement already half-way during the time-range of interest (fig. 8a). The Ne concentration therefore decreases, matching relatively well the CXRS measurement up to $\sim 5(s)$, but going outside of the CXRS confidence interval for later time-points. The match with the bolometer LoS-integrals (fig. $8 \mathrm{~d}$ ) is marginally good, but still worse than case 3. (fig. 6a). Finally, the consistency check that clearly falsifies the assumption that $\mathrm{Ni}$ is the only mid-/high-Z impurity, is the comparison with toroidal rotation (fig. 8k) where it is clear that the asymmetry in the SXR emissivity is far too large to be ascribed to nickel.

\subsection{Impact of other diagnostic uncertainties}

Discrepancies between different diagnostics or LoS measurements are known to take place and it is sometimes unclear which one should be discarded and which one retained for the analysis. In this framework, the main discrepancies that have to be addressed are between LIDAR and HRTS for the electron profile diagnostic and between the vertical and horizontal lines-of-sight of the $Z_{\text {eff }}$ visible Bremmstrahlung measurement.

Using LIDAR instead of HRTS for the electron profile fits (fig. 3c), due to the slightly lower density, a lower background $B e$ concentration $(1.5 \%)$ is necessary to match the 

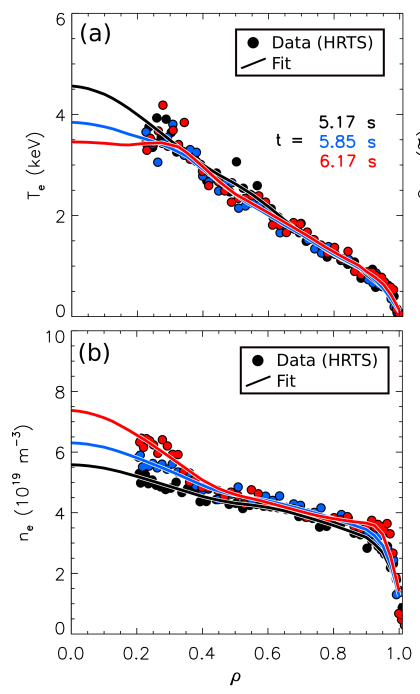

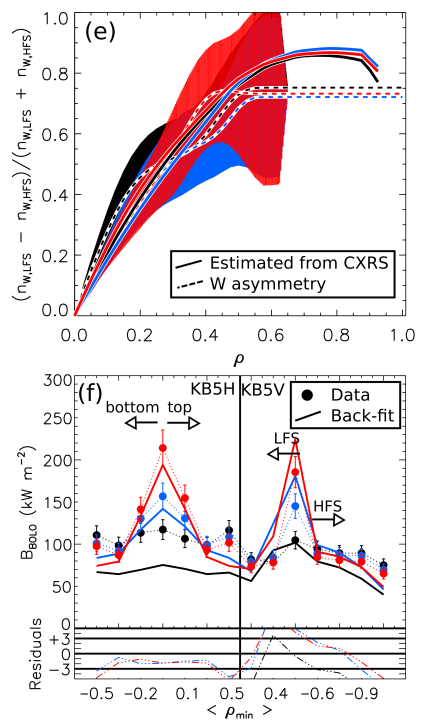

Figure 9. Case 3. analysed using $Z_{\text {eff }}$ measurement $Z E F V$ instead of $Z E F H$. Radial profiles of (a) electron temperature and (b) density (data from HRTS shown as points, fits as continuous lines); time evolution of (c) radially averaged experimental Ne concentration from CXRS (dashed line with confidence interval) compared with the estimated value (continuous line) and (d) flux-surface-averaged $\mathrm{W}$ and $\mathrm{Ni}$ concentrations (continuous and dashed lines respectively) at three different radial positions (colour coded as in label). (e) W density asymmetry (dashed line with confidence band) compared with the estimated value using CXRS measured toroidal rotation; (f) experimental bolometric LoS-integrated measurements (points with error bars) for all LoS shown in fig. 1 compared with the back LoS-integrated values (continuous line).

initial measured $Z_{\text {eff }}$ (still using $Z E F H$ ). With this change only, the final results for Ne, $\mathrm{W}$ and $\mathrm{Ni}$ are all recovered within a few percent and, since the results are well within the final uncertainties, no relevant information can be provided to discard either of the profile diagnostics.

The situation is slightly more complicated when using the vertical LoS in place of the horizontal one (black and red traces in figure 2h). The $Z E F V$ measurement is higher by $\sim 20 \%$ with respect to $Z E F H$ up to around $6 s$ (fig. 2h) and well outside of the statistical uncertainty $(<5 \%)$. In order to match this $Z_{\text {eff }}$ value before the neon puff, a Be concentration of the order of $6 \%$ is necessary. Using this value as a starting point leads to results that are very similar to the original case, for both the $\mathrm{W}$ and $\mathrm{Ni}$ concentrations (fig. 9d) as well as for the $\mathrm{W}$ density asymmetry (fig. 9p) and total radiated power (fig. 9f). The only consistency check that fails dramatically is that with the Ne concentration. The neon required to match the $Z_{\text {eff }}$ measurement is about a factor 2 higher than the one estimated by CXRS and well outside the confidence interval up to $\sim 5.8 \mathrm{~s}$. Beyond this time, when nickel starts contributing substantially to $Z_{\text {eff }}$, the concentration drops going well below the CXRS estimates. This suggests that the vertical measurement is affected by an unknown systematic uncertainty that is not constant in time. As stated earlier in the paper, reflections on the machine wall could lead to an increased value and could be a possible cause of this discrepancy.

These results therefore confirm the choice of $Z E F H$ as the most trustworthy measurement for the effective charge and underline the importance of multiple consistency checks in an integrated framework for the calculation of the plasma composition. If the independent CXRS measurement of the neon concentration were not available, another 
way to assess the reliability of the background Be concentration would be to model the neutron rate (e.g. using TRANSP (Ongena et al. 2012)) which would be affected by the dilution which would increase of $20 \%$ when increasing the Be concentration from 2 to $6 \%$.

\section{Conclusions and outlook}

The methodologies originally developed to model the intrinsic $\mathrm{W}$ density profiles in both ASDEX Upgrade Sertoli et al. (2015) and JET Sertoli et al. (2018) have been further expanded to analyse the plasma composition in JET-ILW including four different impurity species simultaneously: two low-Z and two mid-/high-Z elements. This is necessary because JET-ILW plasmas are known to contain a large zoo of elements originating from the main-chamber walls $(\mathrm{Be})$, the divertor $(\mathrm{W})$, the NBI duct scraper $(\mathrm{Cu})$, the ICRH limiters $(\mathrm{Ni})$ or injected deliberately as seeding gases (Ne, Ar, N, etc.). Such a complex system has an extremely large number of free parameters, but, as demonstrated here, can be relatively well described with a few simple theory-driven assumptions corroborated by experimental evidence, and a complicated, integrated analysis of multiple diagnostic data.

The different behavior of the various elements and their unique contribution to the wide range of measurements included in the analysis is in fact the main reason why they can be disentangled: low-Z elements have low radiated power per unit ion, but contribute strongly to the effective charge; high- $Z$ elements radiate orders of magnitude more, but are minor contributors to $Z_{\text {eff }}$; mid-Z and high-Z elements can be distinguished by their poloidal asymmetries. All of these quantities have to be balanced in order to provide an unambiguous result of the plasma composition.

In the example JET-ILW Ne-seeded hybrid discharge reported here, the observed SXR emission, the $\mathrm{W}$ concentration measured by passive VUV spectroscopy, the LoS $Z_{\text {eff }}$ measurement, the observed poloidal asymmetry of the SXR emission, the CXRS $\mathrm{Ne}$ density and the LoS integrals of the total radiation as measured by bolometry could all be matched including simultaneously $\mathrm{Be}, \mathrm{Ne}, \mathrm{Ni}$ and $\mathrm{W}$. Using a step-wise approach to increase the analysis complexity, attempting to falsify potentially inaccurate measurements, using different background profile diagnostic data and initial assumptions, the final results have been demonstrated to be relatively robust. It has also been proven that data from a few diagnostics is often not enough to distinguish between various possible solutions and can lead to erroneous conclusions which can propagate detrimentally downstream if used for further analysis and modelling.

Of the $\sim 50$ JET-ILW discharges analysed up to now with this method, around 30 have converged to consistent results for all diagnostics. The remaining 20 show good results but do not satisfy all consistency checks simultaneously. The reasons for this could be systematic errors in the measurements (e.g. faults in the data-acquisition) or contamination from unaccounted impurities (e.g. mixed-impurity seeding with $N_{2}$ and $A r)$. Changes in engineering parameters such as variations in gas puff or heating power could also be the cause of varying impurity sources which would lead to inconsistencies in the results with the present assumptions. A temporary and perhaps un-elegant solution would be to analyse such phases separately, trying to match the background conditions as close as possible to the transition.

These limitations suggest possible future enhancements. Information on the sources could be added to avoid the approximation of self-similar and identical time-evolution of the sources. Coupling the analysis with fast transport solvers could give an estimate of the ion temperature screening term in equation 2.5 presently neglected when re-scaling the 
secondary mid-Z impurity density peaking. This coupling would furthermore enable the use of transport-dependent ionization balance and cooling functions. Local-ionizationequilibrium (LIE) used in this publication is known to be trustworthy for mid-/high-Z elements in the plasma core but not in the pedestal region (see e.g. Asmussen et al. (1998); Kallenbach et al. (2013)) where this approximation could lead to underestimate the impurity density and total radiated power (Kallenbach et al. 2013, Fig. 1). If the results of this analysis are to be used in simulations or modeling of particle and heat transport or ELM stability, where the profile shape in the pedestal can change the results dramatically, the confidence in results in the pedestal region has to be increased.

Despite the challenges in accurately determining the plasma composition, these measurements are of prime importance for present-day and future magnetic-controlled-fusion devices with multi-material PFCs. Knowledge of the radial profiles of impurity densities, effective charge and total radiated power are key parameters to correctly understand and model current experiments, and thus also to extrapolate such results to future machines and reactors. Due to the complexity of such measurements, simple assumptions are frequently used to proceed with the transport simulations. These crude approximations can lead to an erroneous calculation of important parameters such as main ion dilution, current density profile and power balance, which propagate further to the estimation of neutron rates, particle and heat transport, turbulence and MHD stability analyses. The methodologies explained in this paper are currently being applied to a variety of discharges to check the entity of these effects and results will be soon reported in a separate publication.

\section{Acknowledgments}

This work has been carried out within the framework of the EUROfusion Consortium and has received funding from the Euratom research and training programme 2014-2018 and 2019-2020 under grant agreement No 633053. The views and opinions expressed herein do not necessarily reflect those of the European Commission

The authors would also like to thank G. Pucella, E. Giovannozzi, C. Bourdelle, C. Maggi, K. Lawson, I. Coffey, T. Pütterich and A. Czarnecka for the fruitful discussions and feedback.

\section{REFERENCES}

Angioni, C., Casson, F. J., Mantica, P., Pütterich, T., Valisa, M., Belli, E. A., Bilato, R., Giroud, C., Helander, P. \& Contributors, J. 2015 The impact of poloidal asymmetries on tungsten transport in the core of JET H-mode plasmas. Physics of Plasmas 22 (5).

Angioni, C., Mantica, P., Pütterich, T., Valisa, M., Baruzzo, M., Belli, E., Belo, P., Casson, F., Challis, C., Drewelow, P., Giroud, C., Hawkes, N., Hender, T., Hobirk, J., Koskela, T., Taroni, L. L., Maggi, C., Mlynar, J., Odstrcil, T., Reinke, M., Romanelli, M. \& JET EFDA Contributors 2014 Tungsten transport in JET H-mode plasmas in hybrid scenario, experimental observations and modelling. Nuclear Fusion $\mathbf{5 4}$ (8), 083028.

Angioni, C., McDermott, R., Fable, E., Fischer, R., Pütterich, T., Ryter, F. \& AND, G. T. 2011 Gyrokinetic modelling of electron and boron density profiles of H-mode plasmas in ASDEX upgrade. Nuclear Fusion 51 (2), 023006.

Asmussen, K., Fournier, K., Laming, J., Neu, R., Seely, J., Dux, R., Engelhardt, W., Fuchs, J. \& ASDEX Upgrade Team 1998 Spectroscopic investigations of tungsten in the EUV region and the determination of its concentration in tokamaks. Nuclear Fusion 38 (7), 967-986. 
Baylor, L. R., Burrell, K. H., Groebner, R. J., Houlberg, W. A., Ernst, D. P., Murakami, M. \& WAde, M. R. 2004 Comparison of toroidal rotation velocities of different impurity ions in the diii-d tokamak. Physics of Plasmas 11 (6), 3100-3105.

Behringer, K., Carolan, P., Denne, B., Decker, G., Engelhardt, W., Forrest, M., Gill, R., Gottardi, N., Hawkes, N., Källne, E., Krause, H., Magyar, G., Mansfield, M., Mast, F., Morgan, P., Peacock, N., Stamp, M. \& Summers, H. 1986 Impurity and radiation studies during the jet ohmic heating phase. Nuclear Fusion 26 (6), 751.

Beurskens, M. N. A., Schweinzer, J., Angioni, C., Burckhart, A., Challis, C. D., Chapman, I., Fischer, R., Flanagan, J., Frassinetti, L., Giroud, C., Hobirk, J., Joffrin, E., Kallenbach, A., Kempenaars, M., Leyland, M., Lomas, P., Maddison, G., Maslov, M., McDermott, R., Neu, R., Nunes, I., Osborne, T., Ryter, F., Saarelma, S., Schneider, P. A., Snyder, P., Tardini, G., Viezzer, E. \& Wolfrum, E. 2013 The effect of a metal wall on confinement in JET and ASDEX Upgrade. Plasma Physics and Controlled Fusion 55 (12), 124043.

Bonanomi, N., Mantica, P., Giroud, C., Angioni, C., Manas, P. \& And, S. M. 2018 Light impurity transport in JET ILW l-mode plasmas. Nuclear Fusion 58 (3), 036009.

Bourdelle, C., Camenen, Y., Citrin, J., Marin, M., Casson, F., Koechl, F. \& And, M. M. 2018 Fast $\mathrm{h}$ isotope and impurity mixing in ion-temperature-gradient turbulence. Nuclear Fusion 58 (7), 076028.

Breton, S., Casson, F., Bourdelle, C., Citrin, J., Baranov, Y., Camenen, Y., Challis, C., Corrigan, G., Garcia, J., Garzotti, L., Henderson, S., Koechl, F., Militello-Asp, E., OMullane, M., Pütterich, T., Sertoli, M. \& and, M. V. 2018 a First principle integrated modeling of multi-channel transport including tungsten in JET. Nuclear Fusion 58 (9), 096003.

Breton, S., Casson, F. J., Bourdelle, C., Angioni, C., Belli, E., Camenen, Y., Citrin, J., Garbet, X., Sarazin, Y. \& Sertoli, M. 2018b High z neoclassical transport: Application and limitation of analytical formulae for modelling JET experimental parameters. Physics of Plasmas 25 (1), 012303, arXiv: https://doi.org/10.1063/1.5019275.

Casson, F. J., Angioni, C., Belli, E. A., Bilato, R., Mantica, P., Odstrcil, T., Pütterich, T., Valisa, M., Garzotti, L., Giroud, C., Hobirk, J., Maggi, C. F., Mlynar, J., Reinke, M. L. \& ASDEX Upgrade Team 2015 Theoretical description of heavy impurity transport and its application to the modelling of tungsten in JET and ASDEX Upgrade. Plasma Physics and Controlled Fusion 57 (1), 014031.

Coenen, J., Sertoli, M., Brezinsek, S., Coffey, I., Dux, R., Giroud, C., Groth, M., Huber, A., Ivanova, D., Krieger, K., Lawson, K., Marsen, S., Meigs, A., Neu, R., Puetterich, T., van RooiJ, G. \& And, M. S. 2013 Long-term evolution of the impurity composition and impurity events with the ITER-like wall at JET. Nuclear Fusion $\mathbf{5 3}$ (7), 073043 .

Czarnecka, A., Zastrow, K.-D., Rzadkiewicz, J., Coffey, I. H., Lawson, K. D. \& and, M. G. O. 2011 Determination of metal impurity density, zeff and dilution on JET by VUV emission spectroscopy. Plasma Physics and Controlled Fusion 53 (3), 035009.

Delgado-Aparicio, L., Stutman, D., Tritz, K., Volpe, F., Wong, K., Bell, R., Finkenthal, M., Fredrickson, E., Gerhardt, S., Kaye, S., LeBlanc, B., Menard, J., PAul, S. \& Roquemore, L. 2011 Impurity transport experiments and effects on MHD in the national spherical torus experiment (NSTX). Nuclear Fusion 51 (8), 083047.

Dux, R. \& Peeters, A. 2000 Neoclassical impurity transport in the core of an ignited tokamak plasma. Nuclear Fusion 40 (10), 1721-1729.

Frassinetti, L., Beurskens, M. N. A., Scannell, R., Osborne, T. H., Flanagan, J., Kempenaars, M., Maslov, M., Pasqualotto, R., Walsh, M. \& Contributors, J. 2012 Spatial resolution of the JET thomson scattering system. Review of Scientific Instruments 83 (1), 013506, arXiv: https://doi.org/10.1063/1.3673467.

Giroud, C., Meigs, A. G., Negus, C. R., Zastrow, K.-D., Biewer, T. M. \& Versloot, T. W. 2008 Impact of calibration technique on measurement accuracy for the jet core charge-exchange system. Review of Scientific Instruments 79 (10), 10F525.

Grierson, B. A., Chrystal, C., Haskey, S. R., Wang, W. X., Rhodes, T. L., McKee, G. R., Barada, K., Yuan, X., Nave, M. F. F., Ashourvan, A. \& Holland, C. 
2019 Main-ion intrinsic toroidal rotation across the itg/tem boundary in diii-d discharges during ohmic and electron cyclotron heating. Physics of Plasmas 26 (4), 042304.

Huber, A., McCormick, K., Andrew, P., Beaumont, P., Dalley, S., Fink, J., Fuchs, J., Fullard, K., Fundamenski, W., Ingesson, L., Mast, F., Jachmich, S., Matthews, G., Mertens, P., Philipps, V., Pitts, R., Sanders, S. \& Zeidner, W. 2007 Upgraded bolometer system on JET for improved radiation measurements. Fusion Engineering and Design 82 (5), 1327 - 1334, proceedings of the 24th Symposium on Fusion Technology.

Ingesson, L., Alper, B., Chen, H., Edwards, A., Fehmers, G., Fuchs, J., Giannella, R., Gill, R., Lauro-Taroni, L. \& Romanelli, M. 1998 Soft x ray tomography during elms and impurity injection in JET. Nuclear Fusion 38 (11), 1675.

Kallenbach, A., Bernert, M., Dux, R., Casali, L., Eich, T., Giannone, L., Herrmann, A., McDermott, R., Mlynek, A., Müller, H. W., Reimold, F., Schweinzer, J., Sertoli, M., Tardini, G., Treutterer, W., Viezzer, E., Wenninger, R. \& and, M. W. 2013 Impurity seeding for tokamak power exhaust: from present devices via ITER to DEMO. Plasma Physics and Controlled Fusion 55 (12), 124041.

Kallenbach, A., Neu, R., Dux, R., Fahrbach, H.-U., Fuchs, J. C., Giannone, L., Gruber, O., Herrmann, A., Lang, P. T., Lipschultz, B., Maggi, C. F., Neuhauser, J., Philipps, V., Pütterich, T., Rohde, V., Roth, J., Sergienko, G., Sips, A. \& ASDEX UpGRAdE TEAM 2005 Tokamak operation with high-zplasma facing components. Plasma Physics and Controlled Fusion 47 (12B), B207-B222.

Kappatou, A., McDermott, R., Angioni, C., Manas, P., Pütterich, T., Dux, R., Viezzer, E., Jaspers, R., Fischer, R., Dunne, M., Cavedon, M., Willensdorfer, M. \& AND, G. T. 2019 Understanding helium transport: experimental and theoretical investigations of low-z impurity transport at ASDEX Upgrade. Nuclear Fusion 59 (5), 056014 .

Lao, L., John, H. S., Stambaugh, R., Kellman, A. \& Pfeiffer, W. 1985 Reconstruction of current profile parameters and plasma shapes in tokamaks. Nuclear Fusion 25 (11), 1611.

LEBSCHY, A. 2018 Experimental characterization of the core plasma flow at the ASDEX Upgrade tokamak. Phd thesis, Technisce Universität München, also IPP report 18/16.

Lerche, E., Eester, D. V., Ongena, J., Mayoral, M.-L., Laxaback, M., Rimini, F., Argouarch, A., Beaumont, P., Blackman, T., Bobkov, V., Brennan, D., Brett, A., Calabro, G., Cecconello, M., Coffey, I., Colas, L., Coyne, A., Crombe, K., Czarnecka, A., Dumont, R., Durodie, F., Felton, R., Frigione, D., Johnson, M. G., Giroud, C., Gorini, G., Graham, M., Hellesen, C., Hellsten, T., Huygen, S., Jacquet, P., Johnson, T., Kiptily, V., Knipe, S., Krasilnikov, A., Lamalle, P., Lennholm, M., Loarte, A., Maggiora, R., Maslov, M., Messiaen, A., Milanesio, D., Monakhov, I., Nightingale, M., Noble, C., Nocente, M., Pangioni, L., Proverbio, I., Sozzi, C., Stamp, M., Studholme, W., Tardocchi, M., Versloot, T. W., Vdovin, V., Vrancken, M., Whitehurst, A., Wooldridge, E. \& And, V. Z. 2011 Optimizing ion-cyclotron resonance frequency heating for ITER: dedicated JET experiments. Plasma Physics and Controlled Fusion 53 (12), 124019.

Loarte, A., Reinke, M. L., Polevoi, A. R., Hosokawa, M., Chilenski, M., Howard, N., Hubbard, A., Hughes, J. W., Rice, J. E., Walk, J., Köchl, F., Pütterich, T., Dux, R. \& Zhogolev, V. E. 2015 Tungsten impurity transport experiments in alcator c-mod to address high priority research and development for iter. Physics of Plasmas $22(5), 056117$.

de la luna, E., Sánchez, J., Tribaldos, V., contributors, J., Conway, G., Suttrop, W., Fessey, J., Prentice, R., Gowers, C. \& Chareau, J. M. 2004 Electron cyclotron emission radiometer upgrade on the joint european torus (JET) tokamak. Review of Scientific Instruments $\mathbf{7 5}$ (10), 3831-3833, arXiv: https://doi.org/10.1063/1.1781376.

Maslov, M., Beurskens, M. N. A., Kempenaars, M. \& Flanagan, J. 2013 Status of the JET LIDAR thomson scattering diagnostic. Journal of Instrumentation 8 (11), C11009C11009.

McDermott, R. M., Dux, R., Pütterich, T., Geiger, B., Kappatou, A., Lebschy, A., Bruhn, C., Cavedon, M., Frank, A., den Harder, N. \& And, E. V. 2018 Evaluation of impurity densities from charge exchange recombination spectroscopy measurements at ASDEX Upgrade. Plasma Physics and Controlled Fusion 60 (9), 095007. 
Menmuir, S., Giroud, C., Biewer, T. M., Coffey, I. H., Delabie, E., Hawkes, N. C., Sertoli, M. \& Contributors, J. 2014 Carbon charge exchange analysis in the iter-like wall environment. Review of Scientific Instruments 85 (11), 11E412, arXiv: https://doi.org/10.1063/1.4890118.

Odstrcil, T., Pütterich, T., Angioni, C., Bilato, R., Gude, A., Odstrcil, M., Team, A. \& THE EUROFUSION MST1 TEAM 2018 The physics of w transport illuminated by recent progress in $\mathrm{w}$ density diagnostics at ASDEX Upgrade. Plasma Physics and Controlled Fusion 60 (1), 014003.

Ongena, J. P. H. E., Voitsekhovitch, I., Evrard, M. \& McCune, D. 2012 Numerical transport codes. Fusion Science and Technology 61 (2T), 180-189.

Pacher, G., Pacher, H., Janeschitz, G., Kukushin, A., Kotov, V. \& Reiter, D. 2007 Modelling of DEMO core plasma consistent with SOL/divertor simulations for long-pulse scenarios with impurity seeding. Nuclear Fusion 47 (5), 469-478.

Pasqualotto, R., Nielsen, P., Gowers, C., Beurskens, M., Kempenaars, M., Carlstrom, T., Johnson, D. \& Contributors, J. 2004 High resolution thomson scattering for joint european torus (JET). Review of Scientific Instruments 75 (10), 38913893, arXiv: https://doi.org/10.1063/1.1787922.

Pucella, G., Giovannozzi, E., Challis, C. D., Chomiczewska, A., Giroud, C., Hobirk, J., Joffrin, E., Kappatou, A., Keeling, D. L., King, D., Krawczyk, N., Lerche, E., Mailloux, J., Menmuir, S., Puetterich, T., Sertoli, M., Voitsekhovitch, I. \& JET CONTRIBUTORs 2019 Evaluation of the effective charge profile and analysis of the effect of the impurity mixture on the current density and safety factor profiles. In $3 r d$ Asia-Pacific Conference on Plasma Physics 2019.

Pütterich, T., Dux, R., Beurskens, M., Bobkov, V., Brezinsek, S., Bucalossi, J., Coenen, J., Coffey, I., Czarnecka, A., Giroud, C., Joffrin, E., Lawson, K., Lehnen, M., de la Luna, E., Mailloux, J., Marsen, S., Mayoral, M.-L., Meigs, A., Neu, R., Rimini, F., Sertoli, M., Stamp, M., van Rooij, G., \& Jet EFdA CONTRIBUTORS 2012 Tungsten screening and impurity control in JET. In Proc. of the 24th IAEA Conference Fusion Energy (CD-Rom), San Diego, USA, October 2012, p. EX/P3-15. Vienna: IAEA.

Pütterich, T., Neu, R., Dux, R., Whiteford, A. D., O’Mullane, M. G. \& ASDEX Upgrade TeAm 2008 Modelling of measured tungsten spectra from ASDEX Upgrade and predictions for iter. Plasma Physics and Controlled Fusion 50 (8), 085016.

Pütterich, T., Fable, E., Dux, R., O’Mullane, M., Neu, R. \& Siccinio, M. 2019 Determination of the tolerable impurity concentrations in a fusion reactor using a consistent set of cooling factors. Nuclear Fusion 59 (5), 056013.

Rathgeber, S. K., Fischer, R., Fietz, S., Hobirk, J., Kallenbach, A., Meister, H., PÜtterich, T., Ryter, F., TARdini, G. \& AND, E. W. 2010 Estimation of profiles of the effective ion charge at ASDEX Upgrade with integrated data analysis. Plasma Physics and Controlled Fusion 52 (9), 095008.

Reinke, M. L., Hutchinson, I. H., Rice, J. E., Howard, N. T., Bader, A., Wukitch, S., Lin, Y., Pace, D. C., Hubbard, A., Hughes, J. W. \& Podpaly, Y. 2012 Poloidal variation of high- $\mathrm{z}$ impurity density due to hydrogen minority ion cyclotron resonance heating on alcator c-mod. Plasma Physics and Controlled Fusion 54 (4), 045004.

Schwob, J. L., Wouters, A. W., Suckewer, S. \& Finkenthal, M. 1987 High-resolution duo-multichannel soft x-ray spectrometer for tokamak plasma diagnostics. Review of Scientific Instruments 58 (9), 1601-1615, arXiv: https://doi.org/10.1063/1.1139408.

Sertoli, M., Angioni, C., Odstrcil, T., ASDeX Upgrade Team \& Team, E. M. 2017 Parametric dependencies of the experimental tungsten transport coefficients in icrh and ecrh assisted ASDEX Upgrade H-modes. Physics of Plasmas 24 (11), 112503, arXiv: https://doi.org/10.1063/1.4996412.

Sertoli, M., Dux, R., Pütterich, T. \& ASDEX Upgrade Team 2015 Modification of impurity transport in the presence of saturated $(\mathrm{m}, \mathrm{n})=(1,1)$ mhd activity at ASDEX Upgrade. Plasma Physics and Controlled Fusion 57 (7), 075004.

Sertoli, M., Flanagan, J., Maslov, M., Maggi, C., Coffey, I., Giroud, C., Menmuir, S., Carvalho, P., Shaw, A., Delabie, E. \& Jet Contributors 2018 Determination of $2 \mathrm{~d}$ poloidal maps of the intrinsic $\mathrm{w}$ density for transport studies in JET-ILW. Review of Scientific Instruments 89 (11), 113501. 
Shumack, A. E., Rzadkiewicz, J., Chernyshova, M., Jakubowska, K., Scholz, M., Byszuk, A., Cieszewski, R., Czarski, T., Dominik, W., Karpinski, L., Kasprowicz, G., Pozniak, K., Wojenski, A., Zabolotny, W., Conway, N. J., Dalley, S., Figueiredo, J., Nakano, T., Tyrrell, S., Zastrow, K.-D. \& Zoita, V. 2014 X-ray crystal spectrometer upgrade for ITER-like wall experiments at JET. Review of Scientific Instruments 85 (11), 11E425, arXiv: https://doi.org/10.1063/1.489118.

Tardini, G., Fischer, R., Jenko, F., Kallenbach, A., McDermott, R. M., Pütterich, T., Rathgeber, S. K., Schneller, M., Schweinzer, J., Sips, A. C. C., Told, D. \& AND, E. W. 2012 Core transport analysis of nitrogen seeded H-mode discharges in the ASDEX Upgrade. Plasma Physics and Controlled Fusion 55 (1), 015010.

Zagórski, R., Ivanova-Stanik, R. \& Stankiewicz, R. 2013 Simulations with the COREDIV code of DEMO discharges. Nuclear Fusion 53 (7), 073030. 